\title{
A comprehensive intervention on feasibility, efficacy, and safety between TAE combined with multi-applicator ablation therapy and TACE in the treatment of large hepatocellular carcinoma
}

\author{
Gehendra Mahara $^{1 \#}$, Guiqun Chen ${ }^{1,2 \#}$, Qi Ge ${ }^{1}$, Zhuochen Lin ${ }^{1}$, Jinhua Huang ${ }^{2}$, Jinxin Zhang ${ }^{1}$ \\ ${ }^{1}$ Department of Medical Statistics and Epidemiology, School of Public Health, Sun Yat-sen University, Guangzhou, China; ${ }^{2}$ Department Minimally \\ Invasive International Therapy, Sun Yat-sen University Cancer Center, Guangzhou, China \\ Contributions: (I) Conception and design: G Mahara, G Chen, Q Ge, J Zhang, J Huang; (II) Administrative support: G Chen, Q Ge, J Zhang; (III) \\ Provision of study materials or patients: G Mahara, G Chen, Q Ge, Z Lin, J Zhang; (IV) Collection and assembly of data: G Mahara, G Chen, Q Ge, \\ J Zhang; (V) Data analysis and interpretation: All authors; (VI) Manuscript writing: All authors; (VII) Final approval of manuscript: All authors. \\ \#These authors contributed equally to this work. \\ Correspondence to: Prof. Jinxin Zhang. Department of Medical Statistics and Epidemiology, School of Public Health, Sun Yat-sen University, \\ Guangzhou, China. Email: zhjinx@mail.sysu.edu.cn; Prof. Jinhua Huang. Department of Minimally Invasive Interventional Therapy, Sun Yat-sen \\ University Cancer Center, No. 651 Dongfeng East Road, Yuexiu District, Guangzhou, China. Email: huangjh@sysucc.org.cn.
}

Background: Hepatocellular carcinoma (HCC) is one of the most common tumors in recent days with high mortality and low early diagnosis rate, resulting in the fourth leading cause of deaths globally. This study aimed to evaluate the feasibility, efficacy, and safety between transcatheter arterial embolization (TAE) therapy followed by multi-applicator ablation and transarterial chemoembolization (TACE) for the treatment of large HCC.

Methods: An intervention study conducted at the Cancer Center of Sun Yat-sen University, Guangzhou, China, with whom had large HCC. A comparison between the two groups (intervention and control group) was evaluated at different time dimensions by repeated-measures analysis of variance, Mann-Whitney $U$ rank-sum test, where Kaplan-Meier and log-rank test calculated for the overall survival (OS), tumor response (TR), and progression-free survival (PFS).

Results: This study found the improved survival rate (SR) and PFS after 1-year treatment in the observation group (Group-I) by $56.4 \%$ and $12.8 \%$, compared to $38.3 \%$ and $8.5 \%$ in the control group (Group-II). Likewise, 1- and 2-year OS rates (OSRs) in Group-I were 66.7\% and 51.4\%, wherein Group-II were $48.9 \%$ and $30.0 \%$, respectively. The short-term efficacy of Group-I, such as complete response (CR), partial response (PR), stable disease (SD), progressive disease (PD) were $28.21 \%, 56.41 \%, 0,15.38 \%$, while in Group-II was 2.13\%, 68.79\%, 6.38\% 22.70\% respectively, which were significant. The short-term efficacy was more excellent in the Group-I than Group-II. The quality of life (QOL) was better in Group-I than that of Group-II.

Conclusions: TAE combined with multi-applicator ablation therapy is safer, effective with prolonged survival, and less severe adverse reactions compared to TACE therapy for the treatment of large HCC.

Keywords: Large hepatocellular carcinoma (large HCC); multi-applicator ablation; transarterial chemoembolization (TACE); transcatheter arterial embolization (TAE); therapy

Submitted Jan 08, 2020. Accepted for publication Sep 12, 2020.

doi: $10.21037 /$ tcr-20-293

View this article at: http://dx.doi.org/10.21037/tcr-20-293 


\section{Introduction}

Hepatocellular carcinoma (HCC) is a common tumor with high mortality and low early diagnosis rate, resulting in the fourth leading causes of deaths globally (1-3). Additionally, delay diagnosis, low success rate, the high recurrence rate after surgery, and poor response rate of medications are generally related to a poor outcome of HCC and becoming a public health burden in the society $(4,5)$. Therefore, the treatment of large HCC is still a challenging job for the clinician (6).

According to the "Guidelines of the diagnosis of primary liver cancer (2011 edition)" (7), a single liver tumor with greater than $>5 \mathrm{~cm}$ diameter is anticipated as a large HCC, which is often diagnosed in the middle and late stages, resulting patients have already lost the best chance of surgery (8). In this stage, only non-surgical treatment can be the option, such as minimally invasive interventional therapies through hepatic artery intubation chemoembolization, transarterial chemoembolization (TACE), transcatheter arterial embolization (TAE), and other ablations therapy. Recent days, TACE and TAE are the most widely used primary therapies for large unresectable HCC (9). The basic principle of TACE and TAE is to cut off the blood supply to the tumor cells and kill the cancerous cells (10).

Several studies have shown that TACE combined with radiofrequency ablation (RFA) or microwave ablation (MWA) was a more effective and significantly prolong the survival time of patients than TACE or ablation alone in the treatment of HCC, especially for large tumor and multiple tumor patients $(9,11)$. However, the retention of TACE chemotherapy drugs has shown severe liver toxicity, as well as affecting the quality of life (QOL) of participants (8). In another side, TAE therapy is better tolerated and slightly safer than TACE, stated by an earlier study (10). Likewise, another study also suggested that HCC may be resistant for chemotherapy; thus, embolization is also important for chemotherapy (12). TAE therapy combined with multiapplicator ablation is completed within a short time (3 days) after hepatic artery embolization $(10,13)$, which is also the less time consuming and low economic burden for the patients and family.

To date, the feasibility, safety, and efficacy of TAE combined with multi-applicator ablation for the treatment of large HCC have not been evaluated broadly. Therefore, this study aimed to appraise the feasibility, effectiveness, and safety of TAE followed by multi-applicator ablation and their clinical application for the treatment of large
HCC. We present the following article in accordance with the STROBE reporting checklist (available at http://dx.doi. org/10.21037/tcr-20-293).

\section{Methods}

\section{Participants}

This cohort study was conducted at the Cancer Center of Sun Yat-sen University, Guangzhou, China, with large HCC patients (age between 18-75 years) from September 2015 to September 2017. This study was conducted in accordance with the Declaration of Helsinki (as revised in 2013). The ethical approval obtained from the Institutional ethical committee of the Cancer Center of Sun Yat-sen University (Approval No. 2016-FXY-021) hospital, and informed consent was taken from all the participants. Group-I received TAE combined with multi-applicator ablation, whereas Group-II received only TACE therapy during the intervention.

The inclusion criteria were: (I) diagnosis is confirmed by imaging (ultrasound/CT/MRI/PET-CT), tumor markers and pathology, the criteria established by the Ministry of Health, "Diagnostic criteria for primary liver cancer" (7); (II) the maximum diameter of the tumor $\geq 5 \mathrm{~cm}$; (III) age between 18 to 75 years; (IV) liver function ChildPugh score grade A or B (score $<7$ points) (Supplement I: Table S1); (V) The ECOG score 0 to 1 (Supplement I: Table S2); (VI) no contraindications to TACE treatment; (VII) no percutaneous radiofrequency RFA or MWA contraindications. And exclusion criteria were: such as (I) HCC combined with severe underlying diseases, poor cardiopulmonary, liver and kidney function, intolerable TACE and ablation therapy; (II) HCC combined with extrahepatic metastases; (III) HCC combined with other parts of the primary tumor; (IV) HCC combined with moderate to severe portal vein or arteriovenous fistula; (V) HCC combined with portal vein tumor thrombus or inferior vena cava tumor thrombus, and (VI) have a history of upper gastrointestinal bleeding.

Equipment for the image guidance, RFA, MWA, and the treatment methods such as TAE, multi-applicator ablation, TACE including equipment for image guidance, RAF, MWA, and C-arm CT guidance, spiral CT guidance method and ablation parameter settings are describing in detail in the Supplement I (Supplementary I). Participant's vital signs, including fasting blood sugar, every 6 hours of blood sugar, and electrocardiogram (ECG) monitored 
until 24 hours after treatment starts. Oxygen concentration was also checked as required. Besides, liver function tests of participants were done regularly, and hemostatic medications were provided as per the need of the participants. Paid attention to the patient's complaint, and given a timely treatment of analgesic, antipyretic, antiemetic medications according to its condition. Antiviral therapy was provided to patients with whom they were positive for hepatitis B surface antigen ( $\mathrm{HBsAg}$ ).

Follow-up: (I) at first and second week after treatment procedure, the following tests were evaluated, such as blood routine, liver function, renal function, coagulation routine, tumor markers, and imaging examination (upper abdomen enhancement MR or CT by Radiologist). Consistently, patients from both groups were followed up in the $1^{\text {st }}$ month, $3^{\text {rd }}$ month, $6^{\text {th }}$ month, $1^{\text {st }}$ year, 1.5 years, $2^{\text {nd }}$ year, $3^{\text {rd }}$ year to both groups. (II) During the follow-up period, participants of both groups were followed-up with treatment therapy such as TACE, ablation, radiotherapy, particle implementation, and target drugs as per the review report. (III) QOL mainly refers to the status of the individual's physiological, psychological, and social functions, which is an important indicator of the effectiveness of the health care services accepted by the patients $(14,15)$. EORTC Quality of Life Measurement Scale QOL-C30 (V3.0) (16) was applied to evaluate the QOL of patients (selected after $1^{\text {st }}$ April, 2017, before treatment, 1, 3, and 6 months after treatment). The Chinese version QOL-C30 (V3.0) is used to collect the data, which is guided directly by the European Organization for Research and Treatment of Cancer (EORTC). Its reliability, validity, and sensitivity has been verified in many countries and successfully applied in clinical research of cancer patients (Supplement I: Supplementary IV) (17).

The overall survival (OS): the time of patients receiving treatment until death or end of the study, was the primary outcome of this study. One-year survival rate (SR) was evaluated after completed a 1-year treatment. Similarly, the progression-free survival (PFS) (the length of time during and an end of treatment of disease progression or occurrence of death). Short-term efficacy was evaluated with the help of enhanced MRI or CT after 1-month treatment using the modified response evaluation criteria for solid tumor (mRECIST standard) $(7,17)$; complete response (CR) (tumor tissue was completely necrotic after treatment for more than 4 weeks, and there was no new lesion). Partial response (PR) (tumor tissue necrosis is more than $30 \%$ for more than 4 weeks after treatment); stable disease (SD) (less than $30 \%$ of the tumor necrosis and no disease progression observed), and progressive disease (PD) (patient developed the disease, and the tumor size increased by more than $20 \%$ ). Safety evaluation (SV): blood routine, liver and kidney function, blood coagulation function, and other indicators were routinely examined before treatment, within 1 week after treatment, and a month after treatment. Local complications were evaluated by the intestine, biliary and gallbladder injury, thoracic and abdominal hemorrhage. Changes in the liver function, kidney function assessed with the systemic complications, and blood coagulation function, as well as symptoms of pain, nausea, and vomiting before and after treatment based on the changes, the result of the above tests.

The sample size was calculated based on the $1^{\text {st }}$ year SR. In this study, we anticipated the $1^{\text {st }}$ year SR of TAE combined with multi-source ablation group was increased by $25 \%$ in comparison to $1^{\text {st }}$ year SR of patients having large HCC treated with TACE alone, which was $28.4 \%$ (18). Power Analysis and Sample Size (PASS) 11.0 tool was used to estimate the sample size. The formula is as follows:

$$
n_{1}=\frac{\left[Z_{\alpha / 2} \sqrt{p(1-p)(1+c) / c}+Z_{\beta} \sqrt{p_{1}\left(1-p_{1}\right)+p_{2}\left(1-p_{2}\right) / c}\right]^{2}}{\left(p_{1}-p_{2}\right)^{2}}
$$

Where, significance level $\alpha=0.05$, power of testing $1-\beta=0.8$. In Group-I assumed SR $P_{1}=0.534$, in Group-II sample rate $\mathrm{P}_{2}=0.284$, sample ratio between two groups $\mathrm{c}=3.5$, combination rate $\mathrm{P}=0.409$. According to the above formula, the required sample size for this study calculated is 37 cases in Group-I and 130 cases in Group-II. In the experiment, some cases might be lost to follow-up. We assumed $10 \%$ for the loss of follow-up. The finally, 39 (among 41 cases, 2 were rejected to participate in the study) in Group-I and 141 cases in Group-II with a total of 180 patients enrolled for the final research.

\section{Statistical analysis}

All data were analyzed and described as the mean, standard deviation, where the $t$-test was applied done to the difference between the two groups' parameters. The indicators of two groups at different time dimensions were compared by repeated measures of analysis of variance ANOVA and Mann-Whitney U rank-sum test. The Pearson chi-square test used for the count data, and Fisher's exact test for categorical data. The OS time and PFS time were calculated by the Kaplan-Meier method, and the log- 
Table 1 Baseline patient's characteristics $(\mathrm{n}=180)$

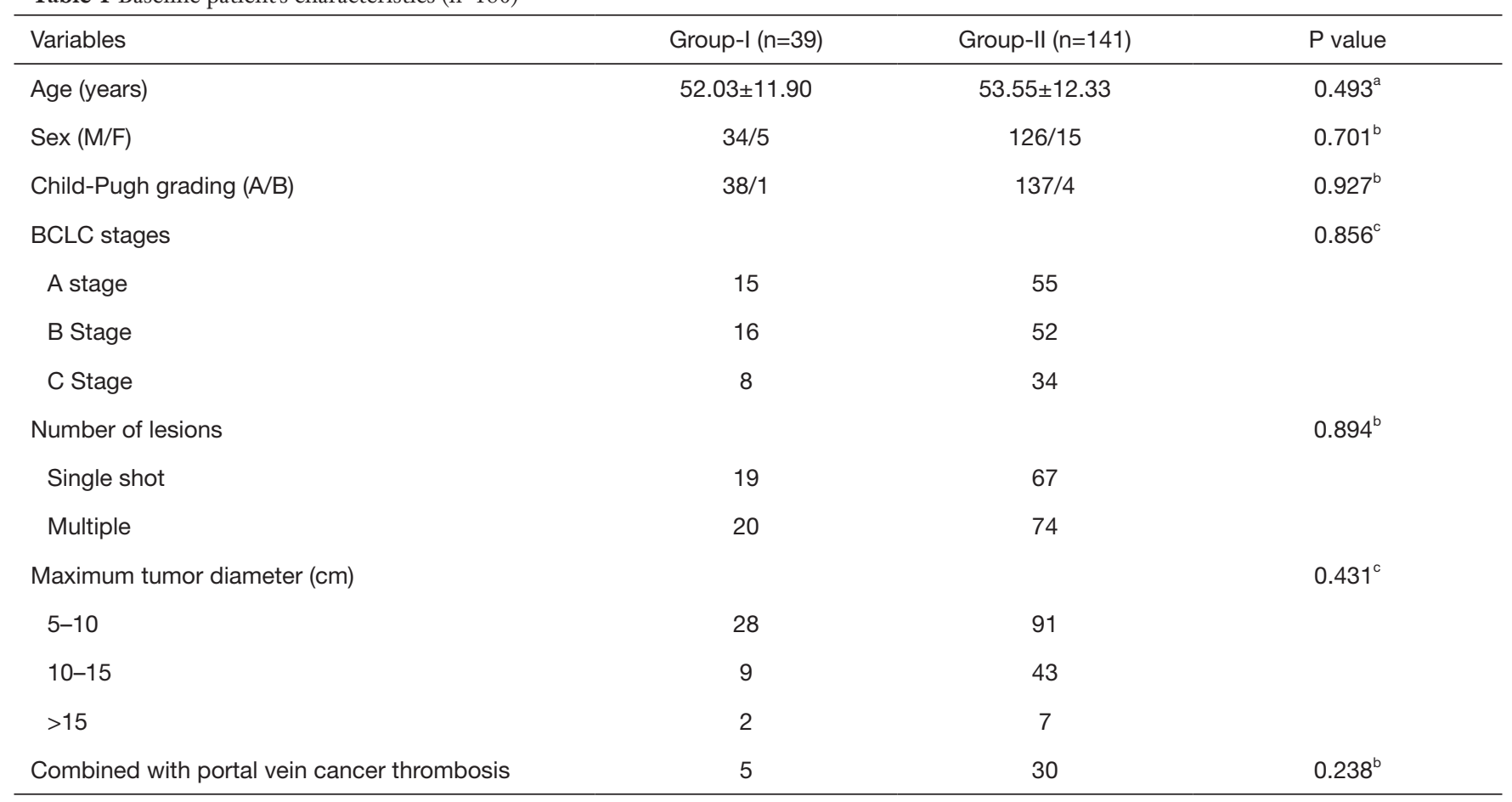

Group-I: Observation Group; Group-II: Control group. ${ }^{a}$, $t$-test; ${ }^{b}$, Pearson chi-square test; ${ }^{\text {, }}$, rank sum test; P<0.05. BCLC, Barcelona Clinic Liver Cancer.

rank test estimated the difference between the two groups. $\mathrm{P}<0.05$ was considered to be statistically significant, and all statistical analysis was performed using SPSS V21.0 software (SPSS Inc., Chicago, IL, USA) (18).

\section{Results}

\section{Basic characteristics}

A total of 180 patients underwent for the intervention, $21.66 \%$ were in the Group-I, and $78.33 \%$ were in the Group-II. Table 1 shows the general information of patients in two groups. There were no significant comparable differences noted in the distribution of age, sex, ChildPugh grading, BCLC stage, number of the lesion, size of the tumor, and thrombosis of the portal vein in both groups (Table 1).

\section{One-year $S R$}

The follow-up period was until September 30, 2018. Among the 39 cases, 22 cases survived until 1 year with a SR of $56.4 \%$ in Group-I, while 54 patients survived with a
$38.3 \%$ SR in Group-II. The $1^{\text {st }}$-year SR of both groups was statistically significant $(\mathrm{P}<0.05)$ (Supplement II: Table S3).

\section{OS rate (OSR)}

The overall result of SR showed that $66.7 \%$ and $51.4 \%$ in the $1^{\text {st }}$ and $2^{\text {nd }}$ year with 15 months median survival time in Group-I while, $48.9 \%$ and $30.0 \%$ with median survival time 12 months in Group-II respectively. The comparison between groups was significant $(\mathrm{P}=0.033)$ by the log-rank test, where OSR was higher in Group-I than that of GroupII ( $\mathrm{P}=0.033)$. Multivariate analysis using Cox regression showed that treatment [hazard ratio $(\mathrm{HR})=0.21$ ], age (HR $=0.98)$, stage $(\mathrm{HR}=2.51)$, and size of the tumor $(\mathrm{HR}=1.97)$ were the influencing risk factors for survival of patients having large HCC. The risk of death in the Group-II was 1.921 times higher than that of the Group-I $(\mathrm{P}<0.05)$ (Figure 1, Table 2).

\section{Progression-free survival}

PFS was evaluated in both groups before the treatment 


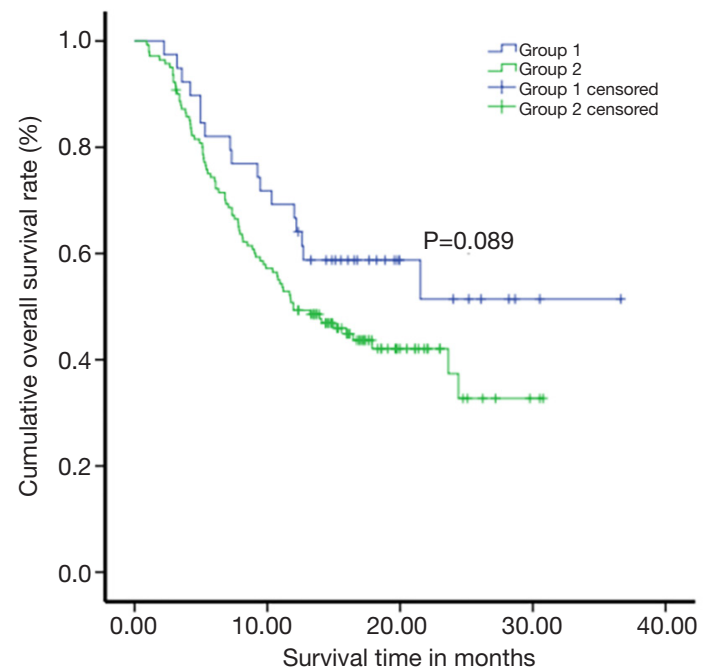

Figure 1 Kaplan-Meier OS of large HCC patients of two groups (Group-I: TAE combined with multi-applicator ablations; Group-II: TACE alone) at different time dimension. OS, overall survival; HCC, hepatocellular carcinoma; TACE, transarterial chemoembolization.

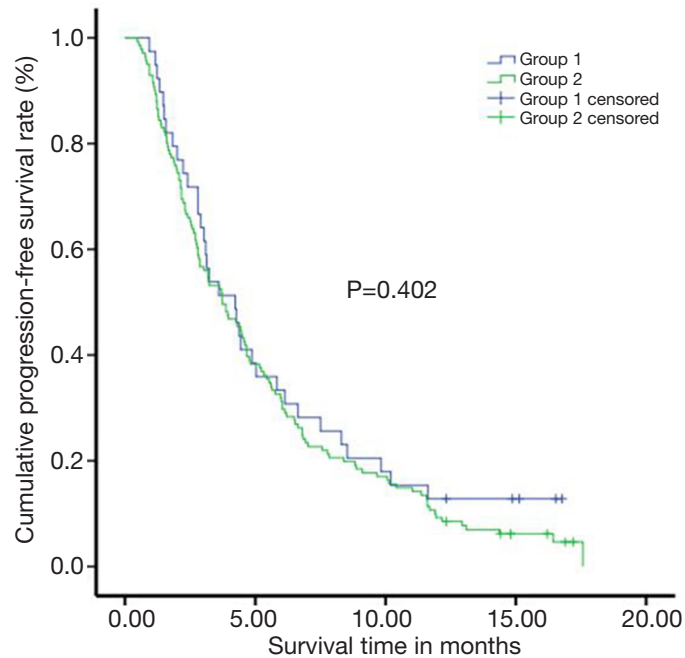

Figure 2 Kaplan-Meier PFS curve of large HCC Patients of two groups (Group-I: TAE combined with multi-applicator ablations; Group-II: TACE alone) at the different time dimension. PFS, progression-free survival; HCC, hepatocellular carcinoma; TACE, transarterial chemoembolization.

Table 2 Multivariate analysis of progression-free risk factors using Cox-regression model

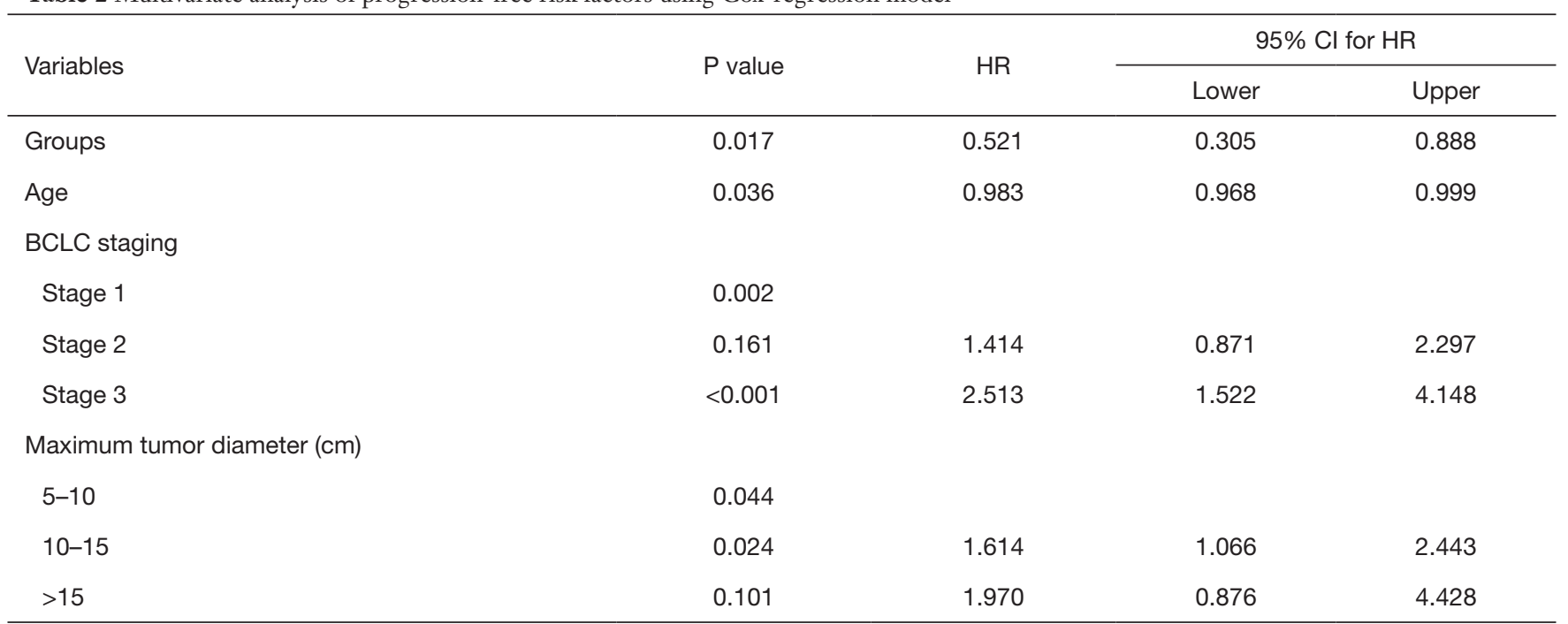

$\mathrm{Cl}$, confidence interval; HR, hazard ratio; BCLC, Barcelona Clinic Liver Cancer. BCLC stage: stage 1, a single tumor of any size, or up to three tumors all less than $3 \mathrm{~cm}$; stage 2, many tumors.

start, and then the progression of the tumor during followup. The enhanced MRI or CT outcomes were assessed as the endpoint of PD and PFS. The PFS was $5.84 \pm 0.79$ months in Group-I, and 5.28 \pm 0.38 months in Group-II. The 1-year PFS rate was $12.8 \%$ in Group-I, and $8.5 \%$ in Group-II. However, there was no significant difference observed in the OFS rate between the two groups $(\mathrm{P}=0.402)$

(Figure 2).

\section{Short-term efficacy}

The short-term efficacy observed after a month of 
Table 3 Comparison of QOL between the two groups before and after treatment

\begin{tabular}{|c|c|c|c|c|c|c|c|c|c|c|c|c|}
\hline Variables & \multicolumn{3}{|c|}{ Before treatment } & \multicolumn{3}{|c|}{$1^{\text {st }}$ month } & \multicolumn{3}{|c|}{$3^{\text {rd }}$ month } & \multicolumn{3}{|c|}{$6^{\text {th }}$ month } \\
\hline F_A & $85.06 \pm 5.64$ & $82.46 \pm 7.41$ & 0.174 & $78.27 \pm 13.10$ & $76.86 \pm 9.47$ & 0.617 & $70.99 \pm 21.44$ & $72.28 \pm 13.73$ & 0.764 & $76.35 \pm 13.49$ & $72.59 \pm 10.57$ & 0.272 \\
\hline S_A & $11.41 \pm 5.56$ & $13.35 \pm 7.31$ & 0.303 & $20.80 \pm 13.07$ & $20.38 \pm 9.61$ & 0.883 & $25.64 \pm 19.07$ & $24.43 \pm 12.60$ & 0.756 & $21.06 \pm 14.16$ & $25.14 \pm 11.50$ & 0.268 \\
\hline O_A & $51.85 \pm 13.87$ & $45.55 \pm 16.76$ & 0.152 & $41.20 \pm 24.00$ & $34.05 \pm 18.16$ & 0.181 & $37.96 \pm 22.36$ & $29.68 \pm 20.29$ & 0.145 & $44.64 \pm 15.20$ & $28.92 \pm 18.13$ & $0.004^{*}$ \\
\hline
\end{tabular}

* indicates $\mathrm{P}<0.05$, a statistically different comparison between the two group. F_A: functional area; S_A: symptom area; O_A: overall area. QOL, quality of life.

treatment, where CR, PR, SD, PD in the Group-I were $28.21 \%, 56.41 \%, 0,15.38 \%$, while Group-II was $2.13 \%$, $68.79 \%, 6.38 \%, 22.70 \%$, respectively. The rank-sum test results showed that the difference in short-term efficacy between the two groups was statistically significant $(\mathrm{z}=-3.525, \mathrm{P}<0.001)$, where Group-I was higher than that of Group-II (Supplement II: Table S4).

\section{Safety evaluation}

After 1 week of treatment, alanine aminotransferase (ALT), aspartate aminotransferase (AST), and total bilirubin (TBiL) increased in both groups, while albumin (ALB) decreased in Group-I $(\mathrm{P}<0.05)$, and creatinine $(\mathrm{CRE})$ remained the normal range, although the difference between the two groups was statistically significant. However, there was no significant difference noted in the above indexes between the two groups after a month of treatment $(\mathrm{P}>0.05)$ (Supplement II: Table S5).

There were significant differences observed in postoperative complications, such as pain, pleural effusion, and biliary tumor between the two groups $(\mathrm{P}<0.05)$; however, no significant difference experienced in both groups such as fever, nausea and vomiting, ascites, hemorrhage, and obstructive jaundice $(\mathrm{P}>0.05)$. These common complications in both groups were relieved after appropriate analgesia, antipyretic, and antiemetic treatment. After treatment, the pleural effusion and ascites were observed in both groups. Two cases of the biliary tumor and one case of obstructive jaundice had improved after treatment, and one case of hemorrhage improved in Group-I after conservative treatment (Supplement II: Table S6).

\section{Quality of life}

QOL evaluated on patients who received treatment after April $1^{\text {st }}, 2017$. There were 18 cases in Group-I and 58 cases in Group-II for the analysis of the QOL. The results showed that there was no significant difference in the QOL between the two groups before treatment, which was comparable. After 1, 3, and 6 months of treatment, the scores of QOL function, symptoms, and overall health pattern scores in both groups met the normal distribution criteria but did not satisfy the testing for spherical symmetry. Multivariate analysis of variance used for the repeated measures. Most of the results were not statistically significant found in both groups. However, the overall health status of patients was found statistically significant in the Group-I and Group-II in the 6 months after treatment $(44.64 \pm 15.20$ vs. $28.92 \pm 18.13, \mathrm{P}<0.05)$ (Table 3).

\section{Discussion}

This study showed that TAE combined with a multiapplicator ablation group, had a better cumulative SR than the single TACE group. OSR $66.7 \%$ and $51.4 \%$ with the 15 months' median survival time noted in Group-I, while $48.9 \%$, and $30 \%$ with 12 months in the simple TACE group in 1- and 2-year after treatment, respectively. Previous studies have shown that combined therapy can prolong the OS of patients compared to simple TACE therapy $(8,9,19)$, which is consistent with our findings. A study of Liu et al. (8), TACE combined with MWA therapy and TACE alone in the treatment of large HCC showed that the median survival time of the two groups was 10 and 6 months, and the average survival time was $(11.61 \pm 1.59)$ and $(6.13 \pm 1.83)$ respectively.

The results of this study showed that the clinical efficacy of TAE combined with multi-applicator ablation for the treatment of large HCC was significantly better-quality by $56.4 \%$ in the intervention group compared with $38.3 \%$ in the Control Group. An earlier study reported that 1-year, the SR of the TACE group was $39.4 \%$ compared to another conservative treatment group (20). This treatment approach 
improved the necrosis rate of patients too. The short-term response was good, and the effect of repeated TACE on the liver was reduced, which is conducive to the recovery of liver function and ultimately showed an excellent longterm SR than TACE alone. Likewise, 1 year SR was higher in Group-I than Group-II, which might be related to the continuous improvement of medical technology and the subsequent taking treatment by patients.

In this study, TAE combined with multi-applicator ablation was used to treat large HCC, where $28.21 \%$, in Group-I vs. $2.13 \%$ in Group-II accomplished during complete inactivation (CR) of large HCC by combination therapy. TAE combined therapy can inactive most or all of the large HCC. There are several theoretical advantages of this therapy stated by many studies (21-24). Multi-applicator ablation requires less time and has a more extensive ablation range compared to single-applicator or multi-point ablation (25). The study of Brace et al. suggested that when using multielectrode ablation at the same time, there may be synergistic effects occurs between adjacent electrodes, which leads to increases in thermal efficiency (26).

During the intervention, the liver function test (ALT, AST, TBiL) increased in both groups after 1 week of treatment, and all the test results of Group-I was higher than that in Group-II, but ALB in the Group-I was lower than Group-II. These findings are similar to previous studies $(9,26,27)$, indicating that a certain degree of liver function damage by the treatment in both groups. Besides, TAE therapy combined with applicator ablation had a more significant impact on liver function. This type of impact on liver function is entirely reversible and controllable by using drugs to protect the liver (28). However, there was no significant difference in the above indexes between two groups after 1 month of treatment, indicating that the impact on liver function caused by treatment is reversible and within a safe range.

Similarly, CRE of both Group increased after a week treatment, wherein Group-II was higher than in the Group-I. TACE may lead to tumor lysis syndrome (28), that is due to the massive release of intercellular substances during treatment, increase the ability of liver metabolism and kidney excretion, which leads to metabolic disorders such as hyperuricemia, hyperkalemia, hyperphosphatemia, and hypocalcemia. Contrary, no patients in Group-I showed tumor lysis syndrome or renal insufficiency, that may occur due to coagulation necrosis, rapid coagulation, degeneration, and inactivation of proteins in tissues and cells (29). Inflammatory factors, allergic factors, and other proteins may remain in cells rather than enter the bloodstream.

The incidence of pain due to TAE combined with multiapplicator ablation was higher in the intervention group (82.05\%), compared to the control group (36.17\%), which is mainly related to the stimulation of hepatic capsule by embolization and ablation agents. During the treatment, the incidence of bile duct injury (two cases: biliary tumors, one case: obstructive jaundice) was noted, mainly manifested as the formation of the biliary tumor and obstructive jaundice. However, these complications can be solved successfully through medical treatment or endoscopic retrograde cholangiopancreatography (ERCP)/percutaneous transhepatic cholangiography and drainage (PTCD).

QOL is a perception of the general well-being of a person or society, in terms of health and happiness, which reflects the concepts of health, mental state, independence level, social relations, and personal beliefs (14). Cancer and its treatment have an impact on all the above areas. Paying attention to the QOL of patients after treatment is a significant development trend in recent years. As a new medical technology, QOL evaluation not only cares about patients' survival time but also pays attention to patient's social and psychological status, patients stress subjective feelings and functional status as well as the health status of individuals or groups from a multi-dimensional perspective (30). TACE therapy has been widely using in the palliative treatment of unresectable HCC. However, this method has a high recurrence rate and significantly affect QOL after treatment. Also, due to ischemia, reperfusion, and retention of chemotherapeutic drugs invade noncancer areas and cause damage to normal liver tissues, which may produce severe hepatotoxicity $(30,31)$. In these circumstances, TAE followed by multi-applicator ablation therapy can be a choice of treatment for large HCC.

A study showed that with the increasing number of TACE therapy that also increases the liver function damage, including the aggravated varying degree of complications, even some patients might die from the deterioration (8). In our study, QOL of Group-II patients gradually decreased after 6 months of treatment. Still, from the 6 months, the overall health indicators, along with the QOL, also started to improve in the Group-I patients, which may be related to the gradual recovery of liver function. Because, after treatment, the first half of the year, all health indicators were increased due to metabolic disorder by the treatment.

This study has several strengths. However, there are some limitations too. First, all therapies had performed 
in the hospital under close supervision, which may lead to inevitable bias due to patient's characteristics, doctor's experience, or equipment quality. Second, some followup treatment may impact the long-term effects of the both methods. Due to the limitations of retrospective studies, it is not clear whether these follow-up treatments differ between the two groups. Third, the sample size of this study was small, and the follow-up time was short. A prospective study with a larger sample size and a more extended followup period is needed to generalize the results more clearly.

\section{Conclusions}

In summary, the feasibility, efficacy, and safety of TAE combined with the applicator ablation are better than that of TACE alone for the treatment of large HCC. The short-term effectiveness and QOL are good, where longterm efficacy can effectively prolong the survival time of patients. Although still need to be further improved, the prolongation of survival time with a relatively high QOL, which brings new hope for patients for large HCC patients who have lost the chance for surgical resection.

\section{Acknowledgments}

The authors thank the Ministry of Science and Technology, National Natural Science Foundation of China and, Natural Science Foundation of Guangdong Province, China, for providing us the fund for this study. The authors would like to acknowledge the patients and their families of study settings and all staff of the Cancer Center of Sun Yatsen University Hospital, Guangzhou, China, for their cooperation, co-ordination, and valuable support.

Funding: This work was supported by the National Natural Science Foundation of China (NSFC, 81773545); and the Natural Science Foundation of Guangdong Province, China (2016A030313365). The funders had no role in study design, data collection, and analysis, decision to publish, or preparation of the manuscript.

\section{Footnote}

Reporting Checklist: The authors have completed the STROBE reporting checklist. Available at http://dx.doi. org/10.21037/tcr-20-293

Data Sharing Statement: Available at http://dx.doi. org/10.21037/tcr-20-293
Peer Review File: Available at http://dx.doi.org/10.21037/tcr20-293

Conflicts of Interest: All authors have completed the ICMJE uniform disclosure form (available at http://dx.doi. org/10.21037/tcr-20-293). The authors have no conflicts of interest to declare.

Etbical Statement: The authors are accountable for all aspects of the work in ensuring that questions related to the accuracy or integrity of any part of the work are appropriately investigated and resolved. This study was conducted in accordance with the Declaration of Helsinki. The ethical approval obtained from the Institutional ethical committee of the Cancer Center of Sun Yat-sen University Hospital (Approval No. 2016-FXY-021), and informed consent was taken from all the participants.

Open Access Statement: This is an Open Access article distributed in accordance with the Creative Commons Attribution-NonCommercial-NoDerivs 4.0 International License (CC BY-NC-ND 4.0), which permits the noncommercial replication and distribution of the article with the strict proviso that no changes or edits are made and the original work is properly cited (including links to both the formal publication through the relevant DOI and the license). See: https://creativecommons.org/licenses/by-nc-nd/4.0/.

\section{References}

1. Llovet JM, Ricci S, Mazzaferro V, et al. Sorafenib in advanced hepatocellular carcinoma. N Engl J Med 2008;359:378-90.

2. World Health Organization. Fact-sheet of Cancer. [cited 2019 Mar 1]. Available online: https://www.who.int/newsroom/fact-sheets/detail/cancer

3. Galun D, Basaric D, Zuvela M, et al. Hepatocellular carcinoma: from clinical practice to evidence-based treatment protocols. World J Hepatol 2015;7:2274-91.

4. Galun D, Bogdanovic A, Kovac Djokic J, et al. Preoperative neutrophil-to-lymphocyte ratio as a prognostic predictor after curative-intent surgery for hepatocellular carcinoma: experience from a developing country. Cancer Manag Res 2018;10:977-88.

5. Torre LA, Siegel RL, Ward EM, et al. Global cancer incidence and mortality rates and trends--an update. Cancer Epidemiol Biomarkers Prev 2016;25:16-27.

6. Zuo TY, Liu FY, Wang MQ, et al. Transcatheter arterial 
chemoembolization combined with simultaneous computed tomography-guided radiofrequency ablation for large hepatocellular carcinomas. Chin Med J (Engl) 2017;130:2666-73.

7. Qin S; Primary Liver Cancer Diagnosis and Treatment Expert Panel of the Chinese Ministry of Health. Guidelines on the diagnosis and treatment of primary liver cancer (2011 edition). Chin Clin Oncol 2012;1:10.

8. Liu C, Liang P, Liu F, et al. MWA combined with TACE as a combined therapy for unresectable largesized hepotocellular carcinoma. Int J Hyperthermia 2011;27:654-62.

9. Chen QW, Ying HF, Gao S, et al. Radiofrequency ablation plus chemoembolization versus radiofrequency ablation alone for hepatocellular carcinoma: a systematic review and meta-analysis. Clin Res Hepatol Gastroenterol 2016;40:309-14.

10. Fiore F, Del Prete M, Franco R, et al. Transarterial embolization (TAE) is equally effective and slightly safer than transarterial chemoembolization (TACE) to manage liver metastases in neuroendocrine tumors. Endocrine 2014;47:177-82.

11. Takaki H, Yamakado K, Uraki J, et al. Radiofrequency ablation combined with chemoembolization for the treatment of hepatocellular carcinomas larger than $5 \mathrm{~cm}$. J Vasc Interv Radiol 2009;20:217-24.

12. Pleguezuelo M, Marelli L, Misseri M, et al. TACE versus TAE as therapy for hepatocellular carcinoma. Expert Rev Anticancer Ther 2008;8:1623-41.

13. Okusaka T, Okada S, Ueno H, et al. Evaluation of the therapeutic effect of transcatheter arterial embolization for hepatocellular carcinoma. Oncology 2000;58:293-9.

14. Collins English Dictionary. Quality of life definition and meaning I Collins English Dictionary. [cited 2019 Apr 5]. Available online: https://www.collinsdictionary.com/ dictionary/english/quality-of-life

15. Olschewski M, Schulgen G, Schumacher M, et al. Quality of life assessment in clinical cancer research. Br J Cancer 1994;70:1-5.

16. Wang J, Chen Z, Lin W, et al. Determination of quality of life of cancer patients in China-EORTC QLQ-C30 trial in China. Acta Psychologica Sinica 2000;32:438-42.

17. Evidence-based practice guidelines for standardized pathological diagnosis of primary liver cancer in China: 2015. Zhonghua Gan Zang Bing Za Zhi 2015;23:321-7.

18. IBM Support. IBM SPSS Statistics 21.0 Available for Download. [cited 2019 Apr 10]. Available online: https:// www-01.ibm.com/support/docview.wss? uid=swg2 1608060
19. Zhang R, Shen L, Zhao L, et al. Combined transarterial chemoembolization and microwave ablation versus transarterial chemoembolization in BCLC stage B hepatocellular carcinoma. Diagn Interv Radiol 2018;24:219-24.

20. Kong JY, Li SM, Fan HY, et al. Transarterial chemoembolization extends long-term survival in patients with unresectable hepatocellular carcinoma. Medicine (Baltimore) 2018;97:e11872.

21. Liang $\mathrm{P}, \mathrm{Yu} \mathrm{J}, \mathrm{Yu} \mathrm{X}$, et al. Microwave ablation for hepatic malignancies. Chinese Journal of Medicine 2015;95:2143-6.

22. Shiraishi R, Yamasaki T, Saeki I, et al. Pilot study of combination therapy with transcatheter arterial infusion chemotherapy using iodized oil and percutaneous radiofrequency ablation during occlusion of hepatic blood flow for hepatocellular carcinoma. Am J Clin Oncol 2008;31:311-6.

23. Dhanasekaran R, Kooby DA, Staley CA, et al. Comparison of conventional transarterial chemoembolization (TACE) and chemoembolization with doxorubicin drug eluting beads (DEB) for unresectable hepatocelluar carcinoma (HCC). J Surg Oncol 2010;101:476-80.

24. Head HW, Dodd GD 3rd, Bao A, et al. Combination radiofrequency ablation and intravenous radiolabeled liposomal Doxorubicin: imaging and quantification of increased drug delivery to tumors. Radiology 2010;255:405-14.

25. Hara AK, Blevins M, Chen MH, et al. ACRIN CT colonography trial: does reader's preference for primary two-dimensional versus primary three-dimensional interpretation affect performance? Radiology 2011;259:435-41.

26. Brace CL, Laeseke PF, Sampson LA, et al. Microwave ablation with multiple simultaneously powered smallgauge triaxial antennas: results from an in vivo swine liver model. Radiology 2007;244:151-6.

27. Díaz-González Á, Reig M, Bruix J. Treatment of hepatocellular carcinoma. Dig Dis 2016;34:597-602.

28. Sakamoto N, Monzawa S, Nagano H, et al. Acute tumor lysis syndrome caused by transcatheter oily chemoembolization in a patient with a large hepatocellular carcinoma. Cardiovasc Intervent Radiol 2007;30:508-11.

29. Luo RG, Fao F, Huang JH, et al. Diluted hydrochloric acid generates larger radiofrequency ablation lesions in excised porcine livers. Diagn Interv Radiol 2013;19:145-9.

30. Peng $\mathrm{P}$, Zheng $\mathrm{Y}$, Wang J. Study on quality of life and its influencing factors in patients with cancer pain. Chinese 
Journal of Oncology 2009;18:276-7.

31. Higuchi T, Kikuchi M, Okazaki M. Hepatocellular carcinoma after transcatheter hepatic arterial embolization.
A histopathologic study of 84 resected cases. Cancer 1994;73:2259-67.

Cite this article as: Mahara G, Chen G, Ge Q, Lin Z, Huang J, Zhang J. A comprehensive intervention on feasibility, efficacy, and safety between TAE combined with multi-applicator ablation therapy and TACE in the treatment of large hepatocellular carcinoma. Transl Cancer Res 2020;9(10):63036312. doi: $10.21037 /$ tcr-20-293 


\section{Supplementary I}

\section{Equipment for the image guidance}

DSA system (Allura Xper FD20, Philips, Netherlands); image post-processing workstation (INTEGRIS 3DRA, Philips, Netherlands); high-pressure injector (Mark V, Medrad, USA); CT equipment (16 rows large) Aperture CT, Philips, The Netherlands).

\section{Equipment for RFA}

Model 1500X radiofrequency therapeutic apparatus; RITA UniBlate monopolar perfusion radiofrequency electrode (RITA Medical Systems, USA). The perfused liquid was diluted by $10 \%$ hydrochloric acid with a perfusion flow rate of $0.2 \mathrm{~mL} / \mathrm{min}$.

\section{Equipment for MWA}

MTC-3C microwave therapeutic instrument; $15 \mathrm{G}$ watercooled circulating microwave antenna (Vision TM Medical, Viking Jiuzhou Medical Instrument, Nanjing, China).

\section{Treatment methods}

Pre-operative improvement of the relevant examinations, including ECG, upper abdominal enhanced CT or MRI, chest X-ray, blood tests (routine blood tests, electrolytes, liver and kidney function, coagulation function, tumor markers, etc.), and need to be tested when the diagnosis is not clear such as Tumor biopsy, PET-CT examination to rule out the distant metastases. Evaluate physical function status, including liver function test with Child-Pugh score and ECOG score. After evaluating the non-therapeutic related contraindications, routine skin preparation and indwelling venous access, an indwelling catheter if necessary, and notify the patients for 12 hours fasting and 6 hours ban water intake before the surgery.

(I) TAE: under local anesthesia, the right femoral artery was punctured percutaneously by the Seldinger method. Routine celiac artery or common hepatic artery angiography was performed with $5 \mathrm{~F}$ YASHIRO or RH catheter, including atrial phase, parenchymal phase, and venous phase, and superior mesenteric artery and iliac artery angiography were performed to identify the collateral blood supply and its movement if necessary. Embolization was performed after $3 \mathrm{~F}$ Terumo or Boston microcatheter was intubated to the targeted angiography of the tumor. Embolization reagents: iodized oil (5-20 mL), gelatin sponge strips, blank microspheres (100-300, 300-500, or 500-700 $\mathrm{m}$ according to tumor staining). The contrast can be seen in the mild dynamic-portal fistula or arteriovenous fistula by the angiography; the fistula should be blocked as much as possible (material: spring coil, gelatin sponge), and then hepatic arterial chemoembolization treatment. If it is difficult to block the fistula orifice, the micro-catheter was inserted to the distal end of the fistula for hepatic artery embolization.

(II) Multi-source ablation: after TAE, percutaneous multi-source MWA or multi-source microwave combined with hydrochloric acid-enhanced RFA (HRFA) were performed simultaneously (within 3 day's interval). An anesthesiologist gave anesthesia during ablation.

\section{Ablation guiding mode and parameter setting}

DSA vascular machine $\mathrm{C}$-arm CT guidance or conventional spiral CT guidance after ablation parameter setting (Supplementary I).

(III) TACE: under local anesthesia, the right femoral artery was punctured percutaneously by the Seldinger method. Celiac artery or common hepatic artery angiography was performed using $5 \mathrm{~F}$ YASHIRO or RH catheter; the imaging images included arterial, parenchymal, and venous phase. The superior mesenteric artery and iliac artery angiography were performed to find out the collateral blood supply artery, the tumor blood supply artery, and its movement. Chemoembolization was performed after $3 \mathrm{~F}$ Termin or Boston micro-catheter incubation to target angiography. Chemotherapy drugs: lobaplatin (30-50 mg), pirarubicin (10-30 mg). Embolization agent: iodized oil $(5-20 \mathrm{~mL})$, gelatin sponge, blank microspheres (100-300, $300-500$, or $500-700 \mu \mathrm{m}$ according to tumor staining). If atrial-portal fistula or atrial-venous fistula is seen on angiography, the fistula orifice blocked as far as possible, and then hepatic artery chemoembolization is performed. If difficult to 
block the fistula orifice, the micro-catheter was inserted to the distal end of the fistula for hepatic arterial chemoembolization.

(IV) Routinely monitored ECG and vital signs for 24 hours after treatment, fasting for 6 hours, and oxygen concentration if necessary. Liver function and hemostatic drugs were given routinely. Paid attention to the patient's complaint, and provided timely treatment of analgesic, antipyretic, antiemetic medications according to its need. Antiviral therapy was given to patients with or without hepatitis B virus activity who were positive for HBsAg.

\section{C-arm CT guidance method}

Due to the limited range of C-arm CT scans, the maximum scan angle is $240^{\circ}$; it cannot reconstruct the complete crosssectional tissue image. Therefore, the patient's right upper abdomen should be placed in the center of the plate to contain the complete tumor tissue. After setting the rotation parameters, rotate the $\mathrm{C}$-arm to the starting position, and perform a rotation scan. The patient was given respiratory training before the operation. The patient remained motionless and held his breath during the CT scan. The patient scanned the image with the liver area as the center and acquired the image. The original image of the $\mathrm{C}$-arm CT was transmitted to the post-processing workstation (Philips Medical Systems INTEGRIS). 3DRA) Image reconstruction of the axial or coronal position as needed. Adjust the puncture path of each needle into the progress position on the operation interface. After the ACC button light flashes, press and hold the button to rotate the C-arm. The position of the ball to reach the angle corresponding to the proposed puncture path. After that, the ACC lamp turns into a long light. According to the position of the C-arm after the rotation is in place, move the infrared cross mark fixed at the end of the bed so that anyone of the marking lines is parallel to the long axis of the C-arm. The other vertical marking line represents the angle of the external needle, and the vertical line is translated. Near the puncture point, guide the needle. When puncturing, the ablation needle is first paralleled with the extracorporeal infrared ray. Then under fluoroscopy, the ablation needle is coincident with the planned puncturing path at the Progress View. After determining the angles of the two directions, the patient is held in a breath; in perspective, Under the dual guidance of the naked eye, the ablation needle is punctured into the intended position. The other operations were repeated for other ablation needle punctures.

\section{Spiral CT guidance method}

Scanning conditions $12 \mathrm{kV}, 265 \mathrm{mS}$, layer thickness $5 \mathrm{~mm}$, pitch $10 \mathrm{~mm}$. According to the location of the patient's tumor, the supine position or prone position is taken. After the metal marking line is attached to the surface of the liver tumor area, a CT scan is performed to determine the acupuncture needle insertion point, the needle insertion depth, and the needle insertion angle. The ablation needle is inserted into the preset ablation needlepoint in the tumor, and after a CT scan confirms the arrival of the site, ablation is started. When the tumor is located in a special position, such as the intestinal tract, diaphragm, stomach and other hollow organs, under the guidance of CT, a chemical ablation needle can be inserted into the gap between the tumor and the intestine to inject physiological saline to separate the tumor tissue from the hollow organ. After the ablation.

\section{Ablation parameter setting}

(I) RFA: power is $100 \mathrm{w}$; time is $20-30$ minutes (single cycle).

(II) MWA: power is $60 \mathrm{w}$, and time is $10-15$ minutes (single cycle).

According to the previous experimental results of isolated bovine liver ablation and related literature: for a mass of 5 to $7 \mathrm{~cm}$ in diameter, use two ablation needles, the maximum distance should not exceed $5 \mathrm{~cm}$; for a tumor with a diameter of 7 to $12 \mathrm{~cm}$, use three ablation needles with the largest distance. Do not exceed $5 \mathrm{~cm}$; a lump with a diameter of $12-15 \mathrm{~cm}$, with four to five ablation needles, the maximum distance should not exceed $6 \mathrm{~cm}$. Avoid the ribs to determine the needle insertion point, puncture path, and needle depth, and ensure that the puncture path avoids important organs such as the intestine and gallbladder. According to the size and shape of the tumor and the actual condition of the needle, if necessary, the needle is ablated. After the ablation, the needle is electro coagulated to avoid bleeding and tumor needle planting. 


\section{Supplementary II}

Table S1 Child-Pugh classification of liver function

\begin{tabular}{lccc}
\hline & \multicolumn{2}{c}{ Scores } \\
\cline { 2 - 3 } Indicators & 1 & 2 & 3 \\
\hline TBiL $(\mu \mathrm{mol} / \mathrm{L})$ & $<34-51$ & $>51$ \\
Serum ALB $(\mathrm{g} / \mathrm{L})$ & $>35$ & $28-35$ & $>28$ \\
Prothrombin time prolong (seconds) & $<4$ & $4-6$ & Moderate and severe \\
Ascites & No & Light & $3-4$ \\
Hepatic encephalopathy (grade) & No & $1-2$ & 3 \\
\hline
\end{tabular}

According to integral method, grade A is $5-6$ points, grade $B$ is $7-9$ points and grade $C$ is $10-15$ points. TBiL, total bilirubin; ALB, albumin.

\section{Supplementary III}

Table S2 ECOG scoring criteria for physical status

\begin{tabular}{ll}
\hline Level & Physical status \\
\hline 0 & The activity and ability was completely normal, and there was no difference from that before onset \\
1 & $\begin{array}{l}\text { Can walk freely and engage in light physical activities, including general housework or office work, but cannot engage in } \\
\text { heavy physical activities }\end{array}$ \\
2 & They can walk freely and take care of themselves, but have lost the ability to work. They can get up at least half of the day \\
3 & Life can only be partially self-care, more than half of the day bed or wheelchair \\
4 & Bed-ridden, life cannot take care of itself \\
\hline
\end{tabular}




\section{Supplementary IV}

\section{EORTC Quality of Life Measurement Scale QLQ-C30 (V3.0)}

We would like to know about you and your health. Please answer all the questions below. The answer here is not "right" or "no". Just ask for the circle that best reflects your situation.

The information you provide will be kept strictly confidential.

Please fill in your code (number):

Date of birth: year-month-day

Today's date: year-month-day

\section{Not quite a bit quite}

1. Are you having trouble with some laborious activities? For example, a heavy shopping bag or suitcase? 1-2-3-4

2. Is it difficult for you to travel long distances? 1-2-3-4

3. Is it difficult for you to walk outdoors for short distances? 1-2-3-4

4. Do you need to stay in bed or chair during the day? 1-2-3-4

5. Do you need someone to help when eating, dressing, bathing or going to the bathroom? 1-2-3-4

\section{In the past week: not a bit quite}

6. Are you restricted in your work and daily activities? 1-2-3-4

7. Are you restricted when engaging in your hobbies or leisure activities? 1-2-3-4

8. Do you have a shortness of breath? 1-2-3-4

9. Do you have pain? $1-2-3-4$

10. Do you need a break? $1-2-3-4$

11. Are you having trouble sleeping? 1-2-3-4

12. Do you feel weak? 1-2-3-4

13. Are you losing your appetite (no appetite)? 1-2-3-4

14. Do you feel sick? $1-2-3-4$

15. Do you have vomiting? $1-2-3-4$

16. Do you have constipation? $1-2-3-4$

17. Do you have diarrhea? 1-2-3-4

18. Do you feel tired? $1-2-3-4$

19. Does pain affect your daily activities? $1-2-3-4$

20. Do you have trouble concentrating on doing things, such as reading a newspaper or watching TV? 1-2-3-4

21. Do you feel nervous? $1-2-3-4$

22. Do you feel worried? $1-2-3-4$

23. Do you feel tempered? $1-2-3-4$

24. Do you feel depressed (low mood)? 1-2-3-4

25. Do you feel that your memory is difficult? $1-2-3-4$

26. Does your physical condition or treatment affect your family life? 1-2-3

27. Does your physical condition or treatment affect your social activities? 1-2-3-4

28. Is your physical condition or treatment making you financially difficult? 1-2-3-4 
For the following questions, please choose the one that best suits you and draw a circle between 1-7

29. How would you rate your overall health over the past week? 1-2-3-4-5-6-7

Very poor, very good

30. How would you rate your total quality of life in the past week? 1-2-3-4-5-6-7

Very poor, very good 


\section{Supplement II}

Table S3 Comparison of 1-year SR in two groups

\begin{tabular}{lcccc}
\hline Parameters & No. of survive & No. of death & Total & SR (\%) \\
\hline Group-I & 22 & 17 & 39 & 56.4 \\
Group-II & 54 & 87 & 141 & 38.3 \\
Total & 76 & 104 & 180 & 42.2 \\
\hline
\end{tabular}

SR, survival rate.

Table S4 Comparison of short-term efficacy between two groups

\begin{tabular}{lcccc}
\hline Parameters & CR & PR & SD & PD \\
\hline Group-I $(\mathrm{n}=39)$ & $11(28.21)$ & $22(56.41)$ & $0(0.00)$ & $6(15.38)$ \\
Group-II $(\mathrm{n}=141)$ & $3(2.13)$ & $97(68.79)$ & $9(6.38)$ & $32(22.70)$ \\
\hline
\end{tabular}

$\mathrm{CR}$, complete response; PR, partial response; SD, stable disease; PD, progressive disease.

Table S5 Changes of blood indexes in the two groups before and after treatment

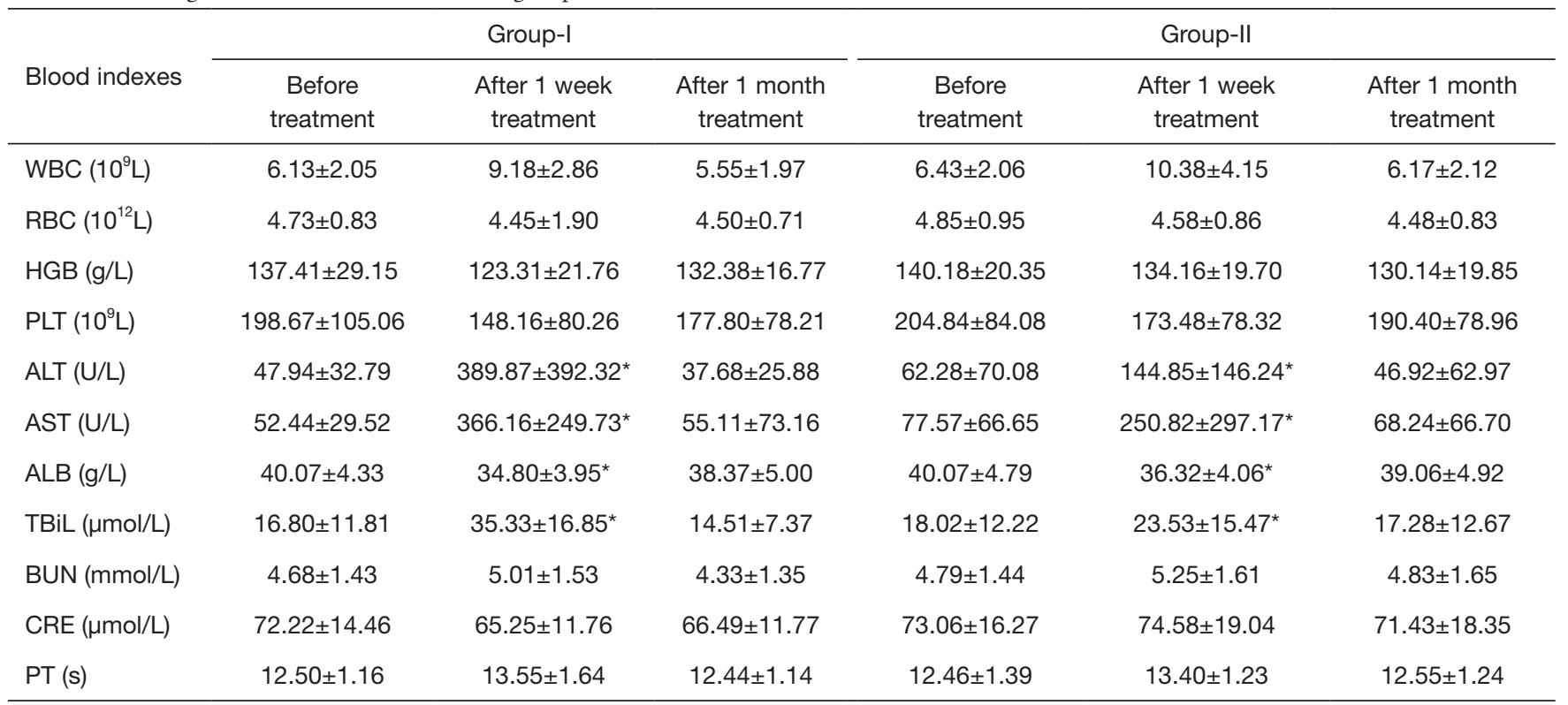

* indicates $\mathrm{P}<0.05$, statistical difference from preoperative comparison. WBC, white blood cells; RBC, red blood cells; HGB, hemoglobin; PLT, platelets; ALT, alanine aminotransferase; AST, aspartate aminotransferase; ALB, albumin; TBiL, total bilirubin; BUN, urea nitrogen; CRE, creatinine; PT, prothrombin time.

Table S6 Comparison of complications between the two groups

\begin{tabular}{|c|c|c|c|c|c|c|c|c|}
\hline Project & Pain & Fever & Nausea \& vomiting & Pleural effusion & Peritoneal effusion & Hemorrhage & Obstructive jaundice & Bile tumor \\
\hline Group-II $(n=141)$ & 51 & 5 & 24 & 1 & 4 & 0 & 0 & 0 \\
\hline$\chi^{2}$ & 25.881 & 0.412 & 0.748 & 17.512 & 0.041 & - & - & - \\
\hline$P$ value & $<0.001^{a *}$ & $0.521^{\text {aa }}$ & $0.387^{\mathrm{a}}$ & $<0.001^{b *}$ & $0.840^{\mathrm{b}}$ & $0.217^{\mathrm{c}}$ & $0.217^{c}$ & $0.046^{\mathrm{c} *}$ \\
\hline
\end{tabular}

\footnotetext{
a , Pearson chi-square test; ${ }^{b}$, continuous calibration chi-square test; ${ }^{c}$, Fisher's exact test. ${ }^{*}$ indicates $\mathrm{P}<0.05$, a statistically significant
} difference between the two group. 